\title{
Hsa_circ_0003159 inhibits gastric cancer progression by regulating miR-223-3p/NDRG1 axis
}

Jingyu Wang ${ }^{1 \dagger}$, Weize $\mathrm{Lv}^{2+}$, Zhidong Lin ${ }^{3}$, Xiao Wang ${ }^{1}$, Juyuan Bu ${ }^{1 *}$ D and Yonghui Su ${ }^{1 *}$

\begin{abstract}
Background: Abnormally expressed circular RNAs (circRNAs) are implicated in the development and treatment of gastric cancer (GC). Previous study has reported that hsa_circ_0003159 is expressed in GC. However, the role and mechanism of hsa_circ_0003159 in GC progression remain unclear.

Methods: GC tissues and normal tissues were harvested from 55 patients in this study. The levels of hsa_ circ_0003159, microRNA (miR)-223-3p and N-myc downstream regulated gene 1 (NDRG1) were measured by quantitative real-time polymerase chain reaction or western blot. Cell proliferation, migration, invasion and apoptosis were determined by cell counting kit (CCK)-8, transwell assay, flow cytometry and western blot, respectively. The target association of miR-223-3p-hsa_circ_0003159 and miR-223-3p-NDRG1 was explored by dual-luciferase reporter assay. Xenograft model was established to assess the roles of hsa_circ_0003159 in GC in vivo.
\end{abstract}

Results: Hsa_circ_0003159 was lowly expressed in GC tissues and cells and mainly presented in the cytoplasm. Low expression of hsa_circ_0003159 was associated with lower overall survival and disease-free survival. Hsa_circ_0003159 overexpression inhibited proliferation, migration and invasion but induced apoptosis in GC cells. MiR-223-3p was a target of hsa_circ_0003159 and abated the effect of hsa_circ_0003159 on proliferation, migration, invasion and apoptosis in GC cells. Hsa_circ_0003159 promoted NDRG1 expression by competitively sponging miR-223-3p. Knockdown of NDRG1 reversed the suppressive effect of hsa_circ_0003159 on GC progression. Besides, hsa_circ_0003159 decreased GC cell xenograft tumor growth by regulating miR-223-3p and NDRG1.

Conclusion: Hsa_circ_0003159 suppressed proliferation, migration, invasion and xenograft tumor growth but promoted apoptosis by decreasing miR-223-3p and increasing NDRG1 in GC, indicating a novel target for treatment of GC.

Keywords: Gastric cancer, hsa_circ_0003159, miR-223-3p, NDRG1, Proliferation, Migration

\section{Background}

Gastric cancer (GC) is one of the most common cancers with a low 5 -year survival rate $(<30 \%)[1,2]$. Recently, the diagnosis and treatment of GC have got significant

\footnotetext{
*Correspondence: ongrunfangchkot@163.com; goubailivknqv@163.com ${ }^{\dagger}$ Jingyu Wang and Weize Lv contributed equally to this work

${ }^{1}$ Department of Gastrointestinal Surgery, The Fifth Affiliated Hospital of Sun Yat-Sen University, No. 52, East Meihua Road, Zhuhai 519000, Guangdong, China

Full list of author information is available at the end of the article
}

progress $[3,4]$. However, the prognosis of GC patients at advanced stages remains unsatisfactory. Therefore, exploring new targets for treatment of GC is excepted at the moment.

Circular RNAs (circRNAs) could act as essential targets for therapeutics of cancers [5]. CircRNAs are a class of noncoding RNAs, which are produced by the backsplicing of a link between upstream splice-acceptor site and downstream splice-donor site [6]. Moreover, circRNAs are widely and stably expressed and

(c) The Author(s) 2020. This article is licensed under a Creative Commons Attribution 4.0 International License, which permits use, sharing, adaptation, distribution and reproduction in any medium or format, as long as you give appropriate credit to the original author(s) and the source, provide a link to the Creative Commons licence, and indicate if changes were made. The images or other third party material in this article are included in the article's Creative Commons licence, unless indicated otherwise in a credit line to the material. If material is not included in the article's Creative Commons licence and your intended use is not permitted by statutory regulation or exceeds the permitted use, you will need to obtain permission directly from the copyright holder. To view a copy of this licence, visit http://creativecommons.org/licenses/by/4.0/. The Creative Commons Public Domain Dedication waiver (http://creativecommons.org/publicdomain/zero/1.0/) applies to the data made available in this article, unless otherwise stated in a credit line to the data. 
take part in the progression of GC [7]. For example, Huang et al. report that circRNA hsa_circ_0008035 could induce tumorigenesis of GC by promoting proliferation and invasion [8]. Furthermore, Liang et al. suggest that hsa_circ_006100 facilitates the tumor growth and metastasis of GC in vitro and in vivo [9]. The hsa_circ_0003159 is arose from CACNA2D1 gene located at chromosome 7:81689743-81746489, which is involved in the development of GC [10]. Nevertheless, the mechanism by which hsa_circ_0003159 mediating $\mathrm{GC}$ progression is largely unknown.

The competing endogenous RNA (ceRNA) hypothesis is an important mechanism allows circRNAs in cancers by sponging microRNAs (miRNAs) to modulate mRNAs [11]. Increasing evidences show that miR-223-3p could participate in regulating cell proliferation, migration and invasion in human cancers, including glioblastomas, renal cell carcinoma and colon cancer [12-14]. Importantly, miR-223-3p has been reported to promote the development of GC by promoting cell proliferation, migration and invasion $[15,16]$. Moreover, $\mathrm{N}$-myc downstream regulated gene 1 (NDRG1) is demonstrated as a metastasis suppressor and inhibit tumor malignancy of GC [17-19]. However, whether miR-223-3p and NDRG1 are associated with the mechanism of hsa_circ_0003159 is unknown. Interestingly, Circular RNA Interactome and starBase online predicted there are the same miR-223-3p binding sites between hsa_circ_0003159 and NDRG1. Hence, we hypothesized that the potential ceRNA network of hsa_circ_0003159/miR-223-3p/NDRG1 might be responsible for the mechanism of hsa_circ_0003159 in GC.

In this study, we detected the expression of hsa circ 0003159 in GC tissues and cell lines and assessed the effect on proliferation, apoptosis, migration, invasion and xenograft tumor growth in GC. Furthermore, this work explored whether hsa_circ_0003159-mediated mechanism was associated with miR-223-3p and NDRG1.

\section{Materials and methods}

\section{Patients and clinical samples}

55 GC patients were recruited from the Fifth Affiliated Hospital of Sun Yat-Sen University and they all signed the written informed consents. The tumor tissues and adjacent normal samples were collected and stored at $-80{ }^{\circ} \mathrm{C}$. The patients' information was shown in Table 1 . The overall survival and disease-free survival were analyzed after a 5-year follow-up. This research has received the approval of the Ethics Committee of the Fifth Affiliated Hospital of Sun Yat-Sen University.
Table 1 Statistics of hsa_circ_0003159 expression in gastric cancer tissues with clinicopathological factors of gastric cancer patients

\begin{tabular}{|c|c|c|c|c|}
\hline \multirow[t]{2}{*}{ Characteristics } & \multirow[t]{2}{*}{$\mathrm{n}$} & \multicolumn{2}{|c|}{ hsa_circ_0003159 } & \multirow[t]{2}{*}{$P$} \\
\hline & & High $(n=27)$ & Low $(n=28)$ & \\
\hline \multicolumn{5}{|l|}{ Gender } \\
\hline Male & 30 & 12 & 18 & \multirow[t]{2}{*}{0.140} \\
\hline Female & 25 & 15 & 10 & \\
\hline \multicolumn{5}{|l|}{ Age (years) } \\
\hline$\geq 60$ & 28 & 13 & 15 & \multirow[t]{2}{*}{0.688} \\
\hline$<60$ & 27 & 14 & 13 & \\
\hline \multicolumn{5}{|l|}{ Tumor size } \\
\hline$\geq 5 \mathrm{~cm}$ & 29 & 11 & 17 & \multirow[t]{2}{*}{0.139} \\
\hline$<5 \mathrm{~cm}$ & 26 & 16 & 11 & \\
\hline \multicolumn{5}{|l|}{ Differentiation } \\
\hline Well + moderate & 20 & 13 & 7 & \multirow[t]{2}{*}{0.074} \\
\hline Poor & 35 & 14 & 21 & \\
\hline \multicolumn{5}{|c|}{ Lymph node metastasis } \\
\hline Yes & 21 & 6 & 15 & \multirow[t]{2}{*}{0.017} \\
\hline No & 34 & 21 & 13 & \\
\hline \multicolumn{5}{|l|}{ Invasion depth } \\
\hline $\mathrm{T} 1+\mathrm{T} 2$ & 19 & 13 & 6 & \multirow[t]{2}{*}{$0.037^{*}$} \\
\hline $\mathrm{T} 3+\mathrm{T} 4$ & 36 & 14 & 22 & \\
\hline \multicolumn{5}{|l|}{ TNM stage } \\
\hline$I+\|$ & 23 & 16 & 7 & \multirow[t]{2}{*}{$0.010^{*}$} \\
\hline$I I I+I V$ & 32 & 11 & 21 & \\
\hline
\end{tabular}

\section{Cell culture}

Human gastric mucosal epithelial cells (GES-1) and GC cell lines (NUGC-3, AGS, HS-746T and N87) were purchased from BeNa Culture Collection (Beijing, China). All cell lines were cultured in Roswell Park Memorial Institute (RPMI)-1640 (Thermo Fisher, Wilmington, DE, USA) containing $10 \%$ fetal bovine serum (Thermo Fisher) and $1 \%$ penicillin-streptomycin solution (Beyotime, Shanghai, China) at $37^{\circ} \mathrm{C}$ in $5 \% \mathrm{CO}_{2}$.

\section{Quantitative real-time polymerase chain reaction (qRT-PCR)}

Total RNA was isolated by Trizol reagent (Solarbio, Beijing, China). The RNA separation of cytoplasmic and nuclear fractions was performed by Cytoplasmic \& Nuclear RNA Purification Kit (Labomics, Nivelles, Belgium). For purity of circRNAs, the RNA was treated by RNase R (Geneseed, Guangzhou, China) for $20 \mathrm{~min}$. $1 \mu \mathrm{g}$ RNA was used for complementary DNA (cDNA) synthesis using the specific TaqMan cDNA synthesis kit (Thermo Fisher). The cDNA products were diluted by $1: 5$ and then mixed with SYBR (Thermo Fisher) and specific primers (Sangon, Shanghai, China) for qRT-PCR. 18S 
rRNA and U6 were regarded as endogenous controls. The primers were listed as: hsa_circ_0003159: Forward, 5'-CCGAACATCTGTCTCCGAAA-3'; Reverse, 5'-CTG CTGCGTGCTGATAAGAT-3'; CACNA2D1: Forward, 5'-CAGTTGAGATGGAGGATGATG-3'; Reverse, 5'-TTGTATGAGCAGTCGTGTGTC-3'; NDRG1: Forward, 5'-ACACCTACCGCCAGCACATT-3'; Reverse, 5'-GGTCGCTCAATCTCCAGGTC-3'; miR-223-3p: Forward, 5'-AGCTGGTGTTGTGAATCAGGCCG-3'; Reverse, 5'-TGGTGTCGTGGAGTCG-3'; 18S rRNA (Forward, 5'-GGCCCTGTAATTGGAATGAGTC-3'; Reverse, 5'-CCAAGATCCAACTACGAGCTT-3'); U6 (Forward, 5'-CTCGCTTCGGCAGCACATATACT-3'; Reverse, 5'-ACGCTTCACGAATTTGCGTGTC-3'). The relative RNA levels were calculated by $2^{-\Delta \Delta C t}$ method [20].

\section{Cell transfection}

The overexpression vector of hsa_circ_0003159 was constructed using pLCDH-ciR vector (Geneseed), with the empty vector (vector) as negative control. The short interfering RNA (siRNA) for hsa_circ_0003159 (si-hsa circ_0003159, 5'-AGCUUCACCUGAGAAAAAUGA$3^{\prime}$ ), siRNA for NDRG1 (si-NDRG1，5'-GCUGAAGCU CGUCAGUUCACCAUCC-3'), siRNA negative control (si-NC, 5'-UUCUCCGAACGUGUCACGU-3'), miR223-3p mimic (miR-223-3p, 5'-UGUCAGUUUGUC AAAUACCCCA-3'), mimic negative control (miR-NC, 5'-GUGGAUUUUCCUCUAUGAUUU-3'), miR-223-3p inhibitor (anti-miR-223-3p, 5'-UGGGGUAUUUGACAA ACUGACA- $3^{\prime}$ ), and inhibitor negative control (anti-NC, $5^{\prime}$-CAGUACUUUUGUGUAGUACAA-3') were synthesized by GenePharma (Shanghai, China). These vectors or oligonucleotides were transfected into NUGC-3 and AGS cells using Lipofectamine ${ }^{\mathrm{TM}} 2000$ transfection reagent (Thermo Fisher) for $24 \mathrm{~h}$.

\section{Cell counting kit (CCK)-8}

NUGC-3 and AGS cells $\left(1 \times 10^{4}\right.$ cells per well) were seeded into 96-well plates and then cultured for 24,48 and $72 \mathrm{~h}$. Next, $10 \mu \mathrm{L}$ of CCK-8 solution (MedChemExpress, Monmouth, NJ, USA) was added to each well and cells were cultured for another $2 \mathrm{~h}$. The optical density value at $450 \mathrm{~nm}$ was detected by a microplate reader (Potenov, Beijing, China).

\section{Flow cytometry}

NUGC- 3 and AGS cells $\left(5 \times 10^{4}\right.$ cells per well) in 12 -well plates were incubated at $37{ }^{\circ} \mathrm{C}$ for $72 \mathrm{~h}$. Subsequently, cells were collected and resuspended in Annexin V-fluorescein isothiocyanate (FITC) binding buffer, followed by incubation of $5 \mu \mathrm{L}$ of Annexin V-FITC and propidium iodide (PI) (Beyotime) for $10 \mathrm{~min}$. The apoptotic cells at upper and lower right quadrants were examined with a flow cytometer (Countstar, Shanghai, China). For cell cycle analysis, NUGC-3 and AGS cells $\left(5 \times 10^{5}\right.$ cells per well) were cultured into 6-well plates for $72 \mathrm{~h}$. Then cells were fixed with $70 \%$ ethanol and incubated with $50 \mu \mathrm{g} /$ $\mathrm{mL}$ PI and RNase A for 20 min. Next, cell cycle distribution was detected via flow cytometer.

\section{Transwell assay}

The abilities of migration and invasion were determined by transwell chambers (Corning, Corning, NY, USA). For migration assay, NUGC-3 and AGS cells $\left(2 \times 10^{5}\right.$ cells $/ \mathrm{mL}$ ) were resuspended in serum-free RPMI-1640 medium and $100 \mu \mathrm{L}$ of cell suspension was plated onto the upper chambers, while $500 \mu \mathrm{L}$ of medium containing $10 \%$ fetal bovine serum was added to the lower chambers. After incubation for $24 \mathrm{~h}$, cells migrated the membranes were fixed and stained with $0.1 \%$ crystal violet (Sigma, St. Louis, MO, USA). Three random fields were selected under the microscope (Olympus, Tokyo, Japan) for counting the number of stained cells. For invasion assay, transwell chambers were precoated with Matrigel, and the procedures were similar with migration assay.

\section{Western blot}

The pre-cold radio immunoprecipitation assay lysis buffer (Beyotime) containing protease inhibitor was used to isolate the total proteins. After the quantification and denaturation, $20 \mu \mathrm{g}$ protein samples were separated by sodium dodecyl sulfate-polyacrylamide gel electrophoresis, and transfected onto polyvinylidene difluoride membranes (Millipore, Billerica, MA, USA). The membranes were interacted with $5 \%$ skim milk for blocking the non-specific sites, and then incubated with primary antibodies at $4{ }^{\circ} \mathrm{C}$ and corresponding secondary antibody. The antibodies were purchased from Abcam (Cambridge, UK) as follows: anti-CyclinD1 (ab226977, 1:2000 dilution), antiCleaved-caspase-3 (ab2302, 1:1000 dilution), anti-matrix metalloproteinase-9 (MMP-9) (ab73734, 1:1000 dilution), anti-NDRG1 (ab196621, 1:2000 dilution), anti-GAPDH (ab37168, 1:2000 dilution) and horseradish peroxidaselabeled IgG (ab205718, 1:10000 dilution). GAPDH was used as an internal control. The blots were visualized by enhanced chemiluminescence reagent (Beyotime) and analyzed by QuantityOne software (Bio-Rad, Hercules, CA, USA).

\section{Dual-luciferase reporter assay}

Circular RNA Interactome and starBase were used to predict the potential targets of hsa_circ_0003159 and miR-223-3p, respectively. The hsa_circ_0003159 sequences and NDRG1 $3^{\prime}$ untranslated region (UTR) containing miR-223-3p binding sites were cloned into 
the downstream of psiCHECK-2 luciferase reporter vector (Promega, Madison, WI, USA) to form the wild-type (WT) luciferase reporter vectors WT hsa_circ_0003159 and NDRG1 3'UTR-WT, respectively. The mutant (MUT) luciferase reporter constructs MUT hsa circ_0003159 and NDRG1 3'UTR-MUT were generated by mutating the binding sites of miR-223-3p. For dualluciferase reporter assay, NUGC-3 and AGS cells were co-transfected with these constructs and miR-223-3p or miR-NC. After $48 \mathrm{~h}$, luciferase activity was measured by a dual-luciferase assay system (Promega).

\section{Xenograft model}

The animal research was conducted under the experimental animal use guidelines and has gained the approval of the ethics committee of the Fifth Affiliated Hospital of Sun Yat-Sen University. Five-week-old male BALB/c nude mice (Charles River, Beijing, China) were used for xenograft model establishment. The lentiviral vector of hsa circ_0003159 was constructed using PLCDH-ciR vector (Geneseed), and the empty vector (vector) was used as negative control. NUGC-3 cells were infected with hsa circ_0003159 or vector, and the stably transfected cells were selected by puromycin. NUGC-3 cells $\left(2 \times 10^{6}\right.$ cells per mouse) stably transfected with hsa_circ_0003159 or vector were subcutaneously injected into the mice $(\mathrm{n}=5$ per group). Tumor grew 28 days and the volume was measured every 7 days, which was calculated with a formula: volume $=$ length $\times$ width $^{2} / 2$. At the ending point, all mice were killed and tumor tissues were weighed. Moreover, the collected tissues were used for hematoxylin-eosin staining or analyses of qRT-PCR and western blot.

\section{Statistical analysis}

The experiment was executed more than 3 times. All data were shown as mean \pm standard deviation (S.D.). GraphPad Prism 7 software (GraphPad Inc., La Jolla, CA, USA) was used for statistical analysis. The overall survival and disease-free survival of GC patients were analyzed via Kaplan-Meier plot and log-rank test. The association between hsa_circ_0003159 expression and clinicopathological factors was analyzed via $\chi^{2}$ test. The independent prognostic factors were analyzed via multivariate analysis. The linear association among the levels of hsa circ_0003159-miR-223-3p, NDRG1-miR-223-3p and NDRG1-hsa_circ_0003159 was analyzed by Spearman's correlation coefficient. Student's $t$ test and one-way analysis of variance were used for the comparison between groups. The difference was considered significant when $P<0.05$.

\section{Results}

Hsa_circ_0003159 expression is reduced in GC

To measure the expression of hsa_circ_0003159 in GC, 55 paired GC tissues and normal samples were collected. The data of qRT-PCR showed that the abundance of hsa_circ_0003159 was significantly decreased in GC tissues when compared to that in normal samples (Fig. 1a). Moreover, the GC patients were divided into high expression group $(n=27)$ and low expression group $(n=28)$ according to the median of hsa_circ_0003159 level. The low expression group displayed lower overall survival $(P=0.031 ; \mathrm{HR}=2.089$ (95\% CI 1.061-4.114)) and disease-free survival $(P=0.0236 ; \quad H R=1.908 \quad(95 \% C I$ 1.059-3.438)) than high expression group after a 5-year follow-up (Fig. 1b, c). Meanwhile, low expression of hsa circ_0003159 was associated with lymph node metastasis, invasion depth and tumor-node-metastasis (TNM) stage (Table 1). Multivariate analysis revealed that lymph node metastasis, invasion depth, TNM stage and low hsa_circ_0003159 were independent risk factors for overall survival of patients (Table 2). In addition, 4 GC cell lines (NUGC-3, AGS, HS-746T and N87) displayed lower level of hsa_circ_0003159 than control GES-1 cells (Fig. 1d). The NUGC-3 and AGS cells with relative lower expression of hsa_circ_0003159 were used for further experiments. Furthermore, hsa_circ_0003159 showed more resistant to RNase $\mathrm{R}$ than corresponding linearCACNA2D1, revealed by the sharp reduction of linear form and few changes of circular form in NUGC-3 and AGS cells (Fig. 1e). Besides, by detecting the abundance of hsa_circ_0003159 in cytoplasm and nuclear, the results showed that this circRNA was mainly localized in cytoplasm (Fig. 1f).

\section{Hsa_circ_0003159 suppresses proliferation, migration and invasion but promotes apoptosis in GC cells}

To explore the role of hsa_circ_0003159 in GC, this circRNA was overexpressed in NUGC-3 and AGS cells by transfection of hsa_circ_0003159 overexpression vector. The transfection efficacy was confirmed by about sixfold increase of hsa_circ_0003159 level in hsa_circ_0003159 group compare with vector group (Fig. 2a, b). Furthermore, the data of CCK-8 assay displayed that overexpression of hsa_circ_0003159 significantly decreased the proliferation of NUGC-3 and AGS cells at $72 \mathrm{~h}$ (Fig. 2c, d). In addition, up-regulation of hsa_circ_0003159 remarkably induced apoptosis production in NUGC-3 and AGS cells at 72 h (Fig. 2e). In addition, overexpression of hsa_circ_0003159 induced cell cycle arrest at G1 phase (Additional file 1: Figure S1). Moreover, the migrated and invasive abilities of NUGC-3 and AGS cells were obviously inhibited by addition of hsa_circ_0003159 at 24 h (Fig. 2f). 


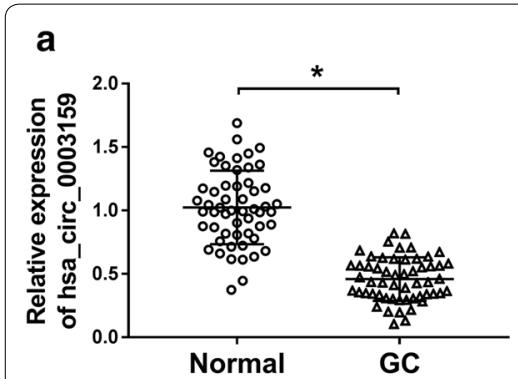

d

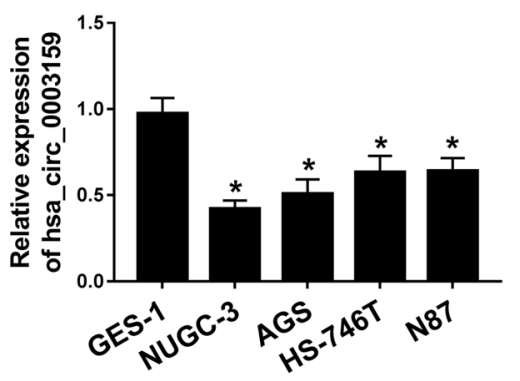

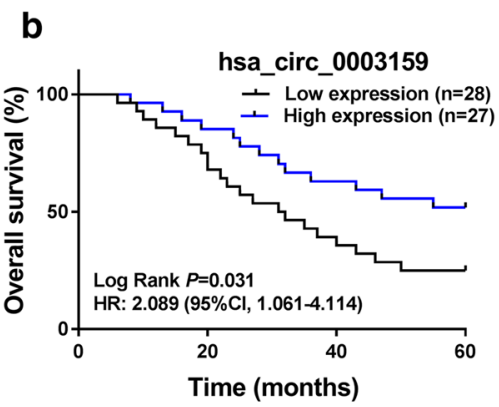

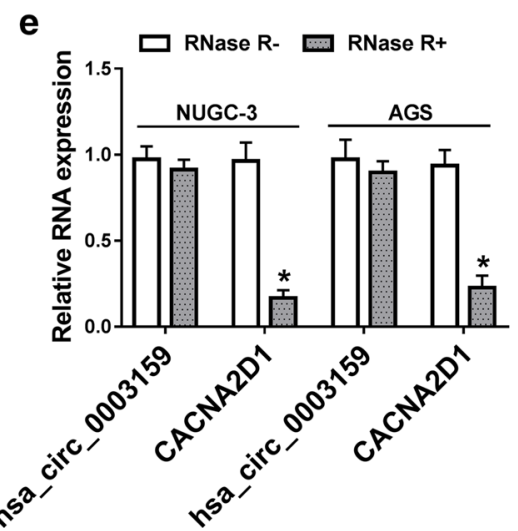

C

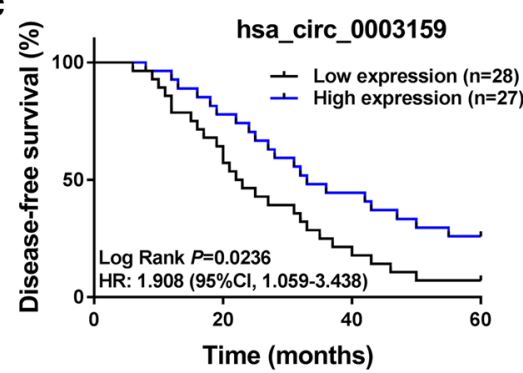

$\mathbf{f}$

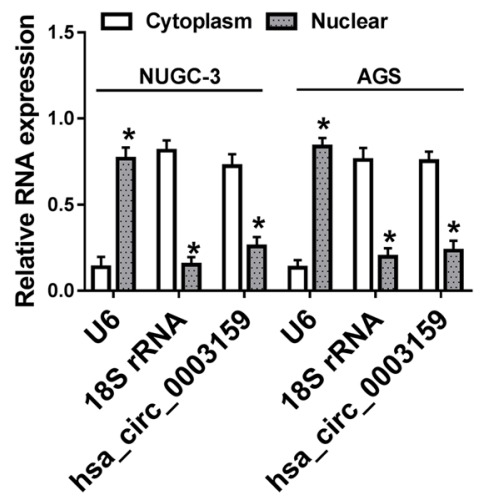

Fig. 1 The expression of hsa_circ_0003159 is decreased in GC. a The expression of hsa_circ_0003159 was detected in GC tissues and normal tissues $(n=55)$ by qRT-PCR. b, c Overall survival (b) and disease-free survival (c) of patients were analyzed according to the expression of hsa_circ_0003159. HR hazards ratio, Cl confidence interval. d The level of hsa_circ_0003159 was detected in GC cell lines (NUGC-3, AGS, HS-746T and N87) and normal GES-1 cells by qRT-PCR. e The expression levels of hsa_circ_0003159 and CACNA2D1 were detected in NUGC-3 and AGS cells after treatment of RNase R or not by qRT-PCR. $\mathbf{f}$ The abundance of hsa_circ_0003159 was examined in NUGC-3 and AGS cells by qRT-PCR, with U6 and 185 rRNA as internal controls for nuclear and cytoplasm, respectively. ${ }^{*} P<0.05$

Table 2 Multivariate analysis of risk factors for survival of patients with gastric cancer

\begin{tabular}{lll}
\hline Characteristics & \multicolumn{2}{l}{ Multivariate analysis } \\
\cline { 2 - 3 } & HR (95\% Cl) & $\boldsymbol{P}$ \\
\hline Lymph node metastasis & $1.85(1.13-3.47)$ & 0.029 \\
Invasion depth & $2.47(1.19-6.39)$ & 0.016 \\
TNM stage & $2.59(1.21-6.51)$ & 0.018 \\
Hsa_circ_0003159 & $0.46(0.32-0.79)$ & 0.011 \\
\hline
\end{tabular}

Cl confidence interval, HR hazard ratio, TNM tumor-node-metastasis

Meanwhile, hsa_circ_0003159 evidently repressed epithelial-mesenchymal transition (Additional file 2: Figure S2). Besides, the protein levels of pro-proliferation CyclinD1, pro-apoptosis Cleaved-caspase-3 and pro-migration MMP-9 were detected in NUGC-3 and AGS cells. Results showed that overexpression of hsa circ_0003159 led to significant reduction of CyclinD1 and MMP-9 and increase of Cleaved-caspase-3 in the two cell lines (Fig. 2g).

\section{Hsa_circ_0003159 acts as a sponge of miR-223-3p in GC} cells

Seeing that hsa_circ_0003159 was mainly located in cytoplasm, the potential miRNAs sponged by hsa circ_0003159 were explored by Circular RNA Interactome. It was predicted that miR-223-3p had the binding sites of hsa_circ_0003159 (Fig. 3a). To validate the association between hsa_circ_0003159 and miR-223-3p, the luciferase reporter vectors WT hsa_circ_0003159 and MUT hsa_circ_0003159 were generated and dual-luciferase reporter assay was performed in NUGC-3 and AGS cells. As shown in Fig. 3b, c, the luciferase activity was declined more than $60 \%$ by miR-223-3p overexpression in WT hsa_circ_0003159 group in the two cell lines, while it showed little effect in MUT hsa_circ_0003159 group. Moreover, the expression of miR-223-3p was markedly enhanced in GC tissues compared with that in normal group (Fig. 3d). Meanwhile, there was an inverse correlation between the levels of hsa_circ_0003159 and miR223-3p in GC tissues $(r=-0.5882, P<0.001)$ (Fig. 3e). NUGC-3 and AGS cells also displayed high expression of miR-223-3p than GES-1 cells (Fig. 3f). In order to 

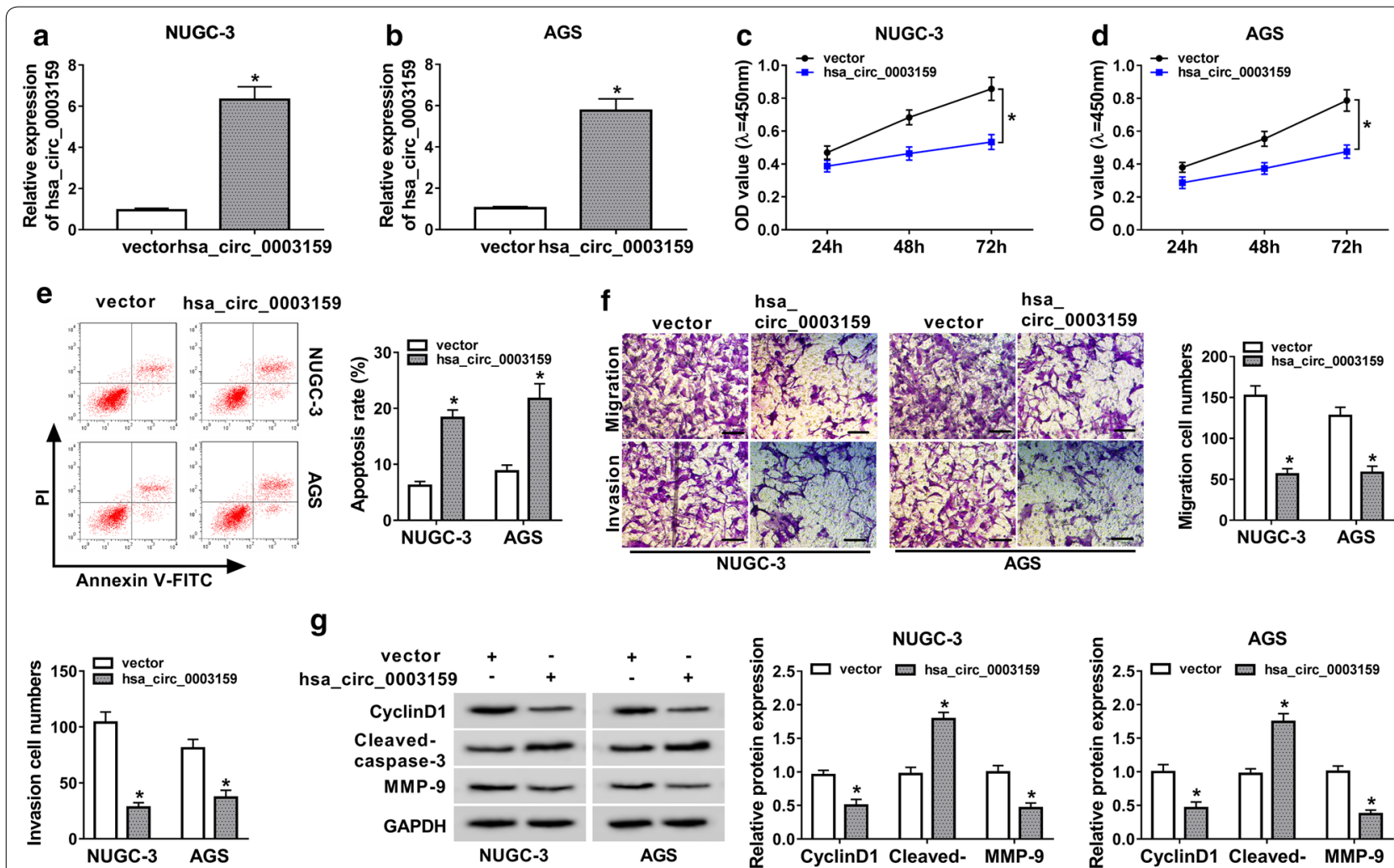

g
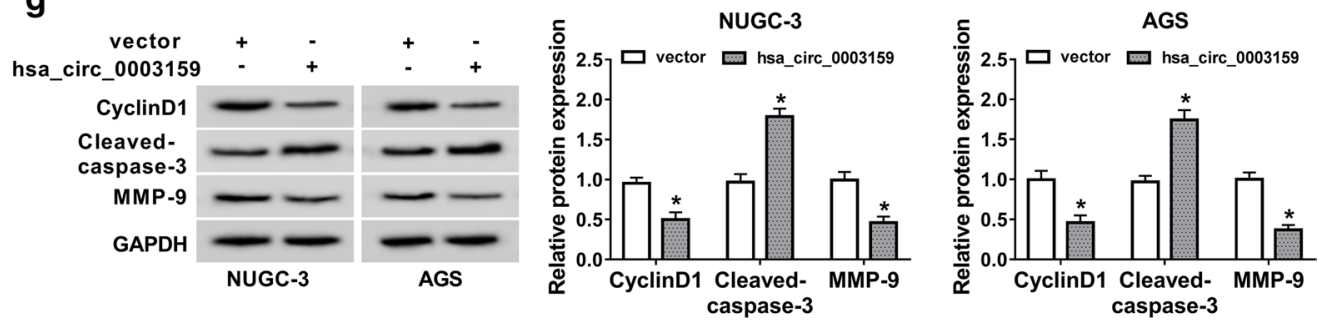

Fig. 2 Overexpression of hsa_circ_0003159 inhibits proliferation, migration and invasion but induces apoptosis in GC cells. a, b The expression of hsa_circ_0003159 was detected in NUGC-3 and AGS cells transfected with hsa_circ_0003159 or vector by qRT-PCR. c, d The proliferation of NUGC-3 and AGS cells transfected with hsa_circ_0003159 or vector was measured at 24, 48 and $72 \mathrm{~h}$ by CCK-8 assay. e The apoptotic rate of NUGC-3 and AGS cells transfected with hsa_circ_0003159 or vector was detected at $72 \mathrm{~h}$ by flow cytometry. $\mathbf{f}$ The migrated and invasive abilities of NUGC-3 and AGS cells transfected with hsa_circ_0003159 or vector were analyzed at $24 \mathrm{~h}$ by transwell assay. Scale bar: $50 \mu \mathrm{m}$. $\mathbf{g}$ The protein levels of CyclinD1, Cleaved-caspase-3 and MMP-9 were measured in NUGC-3 and AGS cells transfected with hsa_circ_0003159 or vector at $72 \mathrm{~h}$ by western blot. ${ }^{*} P<0.05$

investigate the effect of hsa_circ_0003159 on miR-223-3p expression, NUGC-3 and AGS cells were transfected with vector, hsa_circ_0003159, si-NC or si-hsa_circ_0003159. The knockdown efficacy of hsa_circ_0003159 was validated in Fig. 3g. Additionally, miR-223-3p expression in NUGC-3 and AGS cells was evidently decreased by hsa_circ_0003159 overexpression and increased by hsa circ_0003159 knockdown (Fig. 3h, i).

\section{Overexpression of miR-223-3p attenuates the effect} of hsa_circ_0003159 on proliferation, apoptosis, migration and invasion in GC cells

The transfection efficacy of miR-223-3p and anti-miR223-3p was confirmed in Fig. 4a. To explore whether miR-223-3p was required for hsa_circ_0003159-meidated mechanism, NUGC-3 and AGS cells were transfected with vector, hsa_circ_0003159, hsa_circ_0003159 and miR-NC or miR-223-3p mimic. As shown in Fig. 4b, $c$, the up-regulation of miR-223-3p abated the suppressive role of hsa_circ_0003159 in proliferation of NUGC-3 and AGS cells. Moreover, miR-223-3p addition mitigated cell apoptosis induced by hsa_circ_0003159 overexpression in the two cell lines (Fig. 4d, e). Additionally, the impaired abilities of migration and invasion induced by hsa_circ_0003159 was restored by miR-223-3p overexpression in NUGC-3 and AGS cells (Fig. 4f-h). Besides, the regulatory effect of hsa_circ_0003159 on CyclinD1, Cleaved-caspase-3 and MMP-9 protein levels was weakened by miR-223-3p overexpression (Fig. 4i).

\section{Hsa_circ_0003159 promotes NDRG1 expression by regulating miR-223-3p in GC cells}

To further elucidate the ceRNA network mediated by hsa_circ_0003159, the target s of miR-223-3p were explored by starBase, which showed that NDRG1 had the miR-223-3p binding sites (Fig. 5a). For validation of the target association between miR-223-3p and NDRG1, the luciferase reporter vectors NDRG1 3'UTRWT and NDRG1 $3^{\prime} U T R-M U T$ were constructed and transfected into NUGC-3 and AGS cells. The analysis 


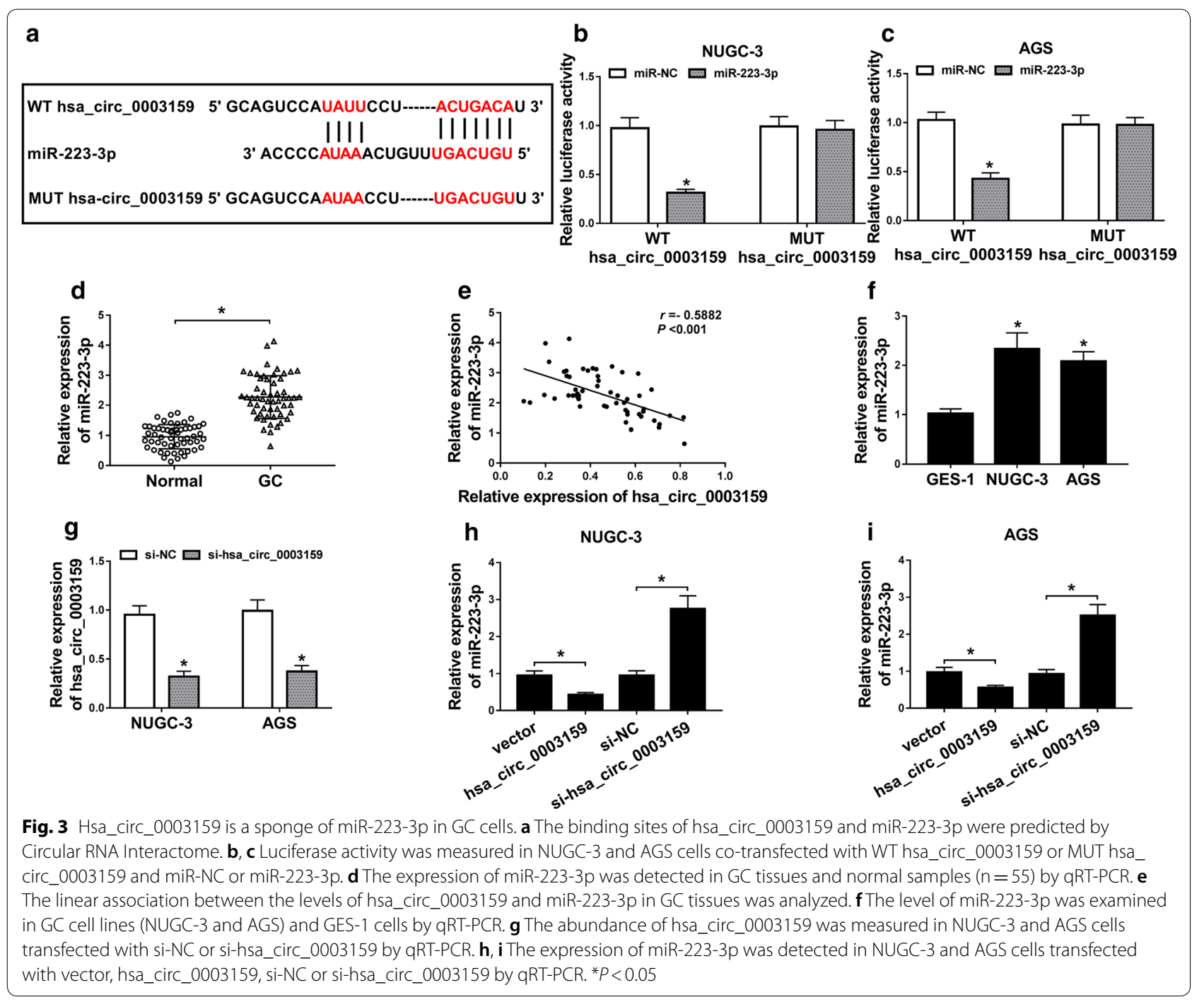

of dual-luciferase reporter described that miR-223-3p overexpression led to great loss of luciferase activity in NDRG1 3'UTR-WT group, whereas it did not affect the activity in NDRG1 $3^{\prime}$ UTR-MUT group (Fig. 5b, c). Moreover, the mRNA and protein levels of NDRG1 were significantly reduced in GC tissues compared with those in normal samples (Fig. 5d, e). Meanwhile, the mRNA level of NDRG1 was negatively associated with miR-223-3p level $(r=-0.468, P=0.0003)$ and positively corelated with hsa_circ_0003159 expression $(\mathrm{r}=0.3887$, $P=0.0034$ ) in GC tissues (Fig. 5f, g). In addition, the expression of NDRG1 was also evidently decreased at transcriptional and protein levels in NUGC-3 and AGS cells than that in GES-1 cells (Fig. 5h, i). Furthermore, the abundances of NDRG1 mRNA and protein in NUGC-3 and AGS cells were significantly elevated by miR-223-3p knockdown (Fig. 5j, k). Besides, the mRNA and protein levels of NDRG1 were greatly enhanced by hsa_circ_0003159 overexpression, which was weakened by introduction of miR-223-3p in NUGC-3 and AGS cells (Fig. 5l, m).

Silence of NDRG1 alleviates the effect of has_circ_0003159 on proliferation, migration, invasion and apoptosis in GC cells

To explore whether hsa_circ_0003159-regulated GC progression was mediated by NDRG1, NUGC-3 and AGS cells were transfected with vector, hsa circ_0003159, hsa_circ_0003159 and si-NC or siNDRG1. The suppressive efficacy of si-NDRG1 on NDRG1 expression in NUGC-3 and AGS cells was confirmed by sharply decreased NDRG1 in si-NDRG1 group (Fig. 6a, b). Furthermore, silence of NDRG1 abated the suppressive effect of hsa_circ_0003159 on proliferation of NUGC-3 and AGS cells (Fig. 6c, d). In addition, hsa_circ_0003159-induced apoptosis 

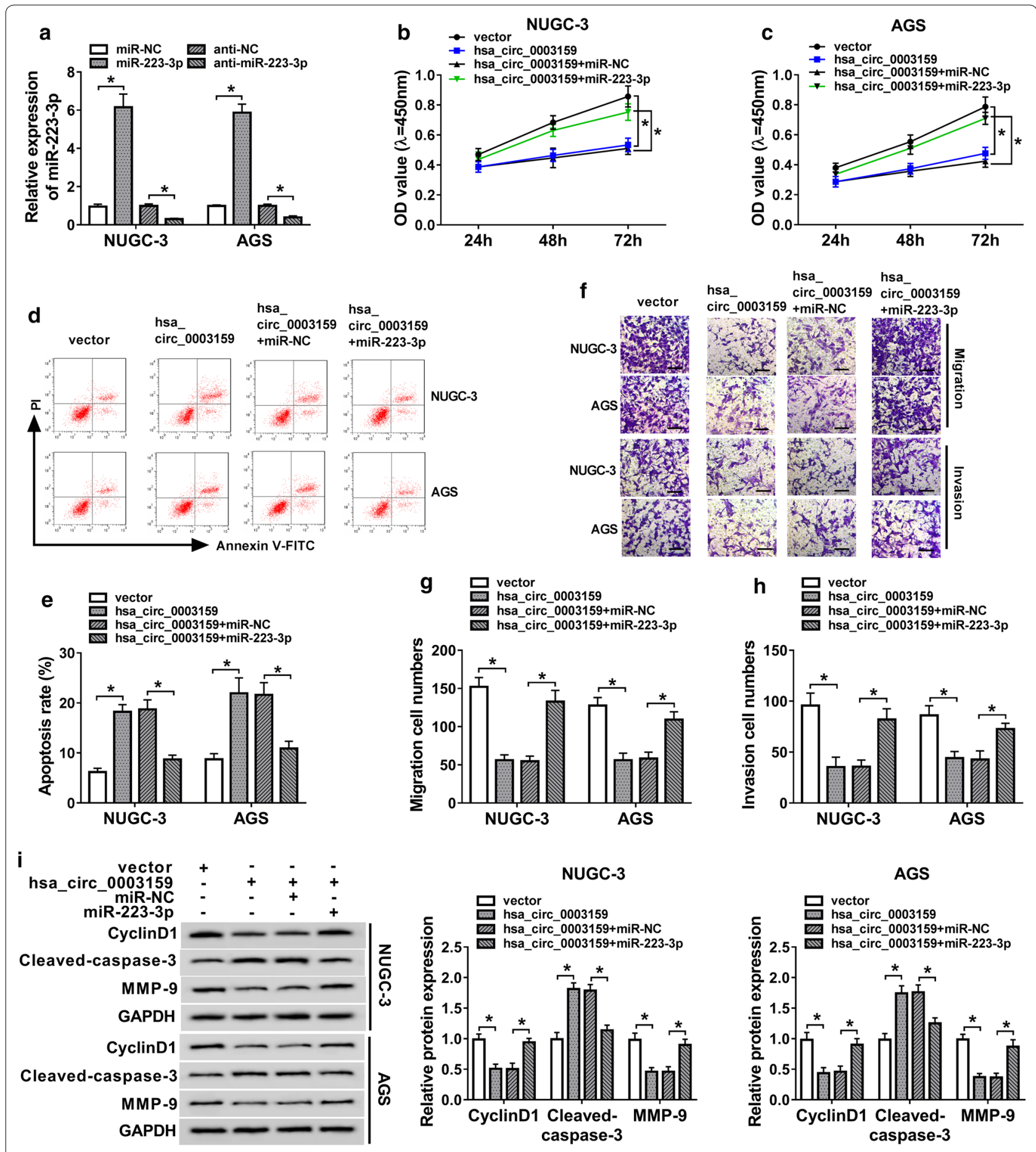

Fig. 4 Up-regulation of miR-223-3p reverses the effect of hsa_circ_0003159 on proliferation, migration, invasion and apoptosis in GC cells. a The expression of miR-223-3p was measured in NUGC-3 and AGS cells transfected with miR-NC, miR-223-3p, anti-NC or anti-miR-223-3p by qRT-PCR. b-g Cell proliferation $(\mathbf{b}, \mathbf{c})$, apoptosis $(\mathbf{d}, \mathbf{e})$, migration and invasion $(\mathbf{f}-\mathbf{h})$ and protein levels of CyclinD1, Cleaved-caspase-3 and MMP-9 (i) were detected in NUGC-3 and AGS cells transfected with vector, hsa_circ_0003159, hsa_circ_0003159 and miR-NC or miR-223-3p by CCK-8, flow cytometry, transwell and western blot assays, respectively. ${ }^{*} P<0.05$ 


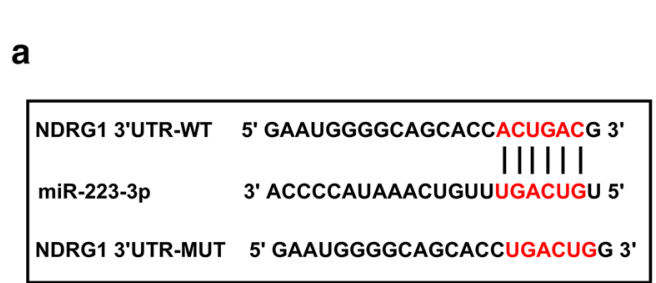

d

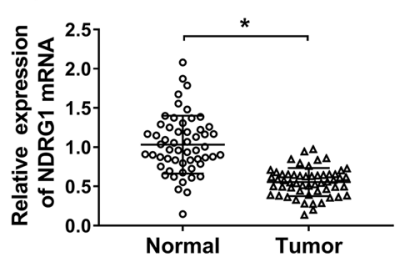

h

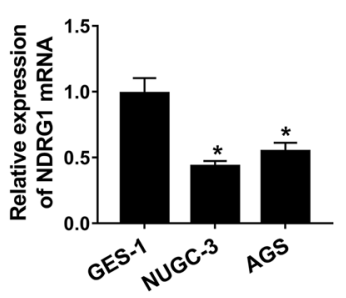

I

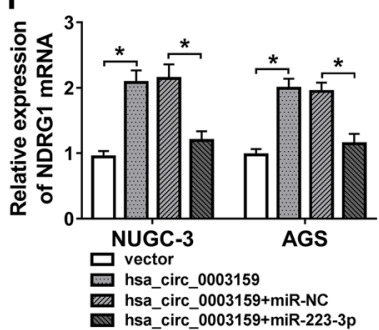

b

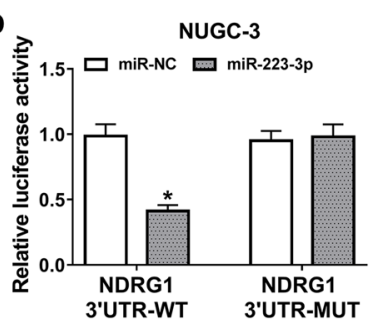

e
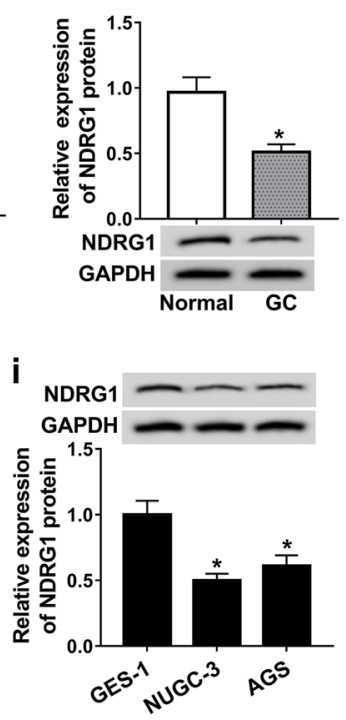

f

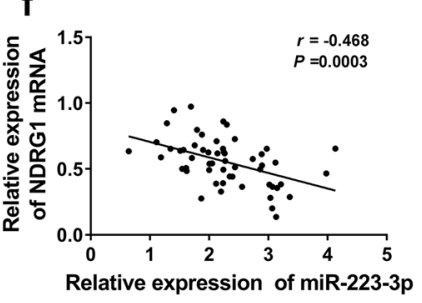

j

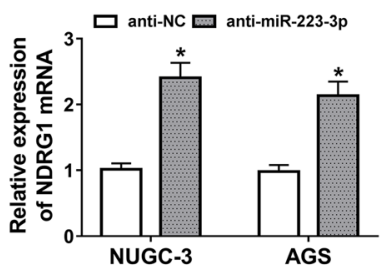

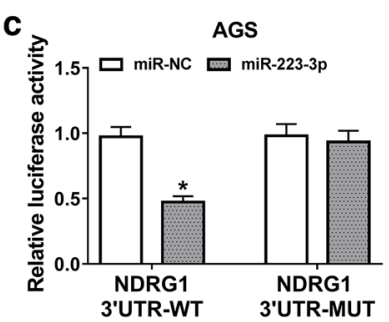

g

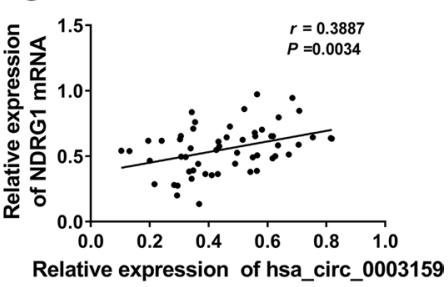

k
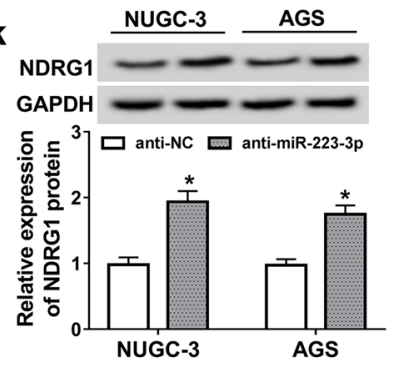

m
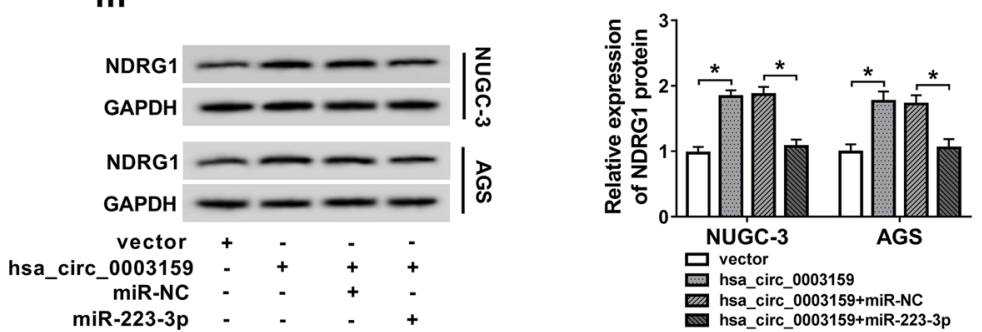

Fig. 5 NDRG1 is regulated by hsa_circ_0003159 and miR-223-3p in GC cells. a The binding sites of miR-223-3p and NDRG1 were predicted by starBase. b, c Luciferase activity was measured in NUGC-3 and AGS cells co-transfected with NDRG1 3'UTR-WT or NDRG1 3'UTR-MUT and miR-NC or miR-223-3p. d, e The expression levels of NDRG1 mRNA and protein were detected in GC tissues and normal tissues by qRT-PCR and western blot. $\mathbf{f}, \mathbf{g}$ The association between expression of NDRG1 mRNA and miR-223-3p or hsa_circ_0003159 in GC tissues was assessed. $\mathbf{h}, \mathbf{i}$ The mRNA and protein levels of NDRG1 were measured in GC cells (NUGC-3 and AGS) and GES-1 cells by qRT-PCR and western blot. $\mathbf{j}, \mathbf{k}$ The mRNA and protein levels of NDRG1 were measured in NUGC-3 and AGS cells transfected with anti-miR-223-3p or anti-NC by qRT-PCR and western blot. $\mathbf{l}$, $\mathbf{m}$ The $\mathbf{m R N A}$ and protein levels of NDRG1 were examined in NUGC-3 and AGS cells transfected with vector, hsa_circ_0003159, hsa_circ_0003159 and miR-NC or miR-223-3p by qRT-PCR and western blot. ${ }^{*} P<0.05$

was weakened by down-regulation of NDRG1 in NUGC-3 and AGS cells (Fig. 6e). Moreover, interference of NDRG1 abrogated the inhibitive effect of hsa circ_0003159 on migration and invasion of NUGC-3 and AGS cells (Fig. 6f, g). Besides, the reduction of CyclinD1 and MMP-9 and increase of Cleaved-caspase-3 mediated by hsa_circ_0003159 were reversed by NDRG1 silence in the two cell lines (Fig. 6h, i).
Hsa_circ_0003159 reduces GC cell xenograft tumor growth To further investigate the effect of hsa_circ_0003159 on GC progression in vivo, NUGC-3 cells stably transfected with vector or hsa_circ_0003159 were subcutaneously injected into nude mice, named as vector or hsa_circ_0003159 group, respectively. The tumor volume was significantly decreased at 21 and 28 days in hsa circ_0003159 group compared with that in vector group 


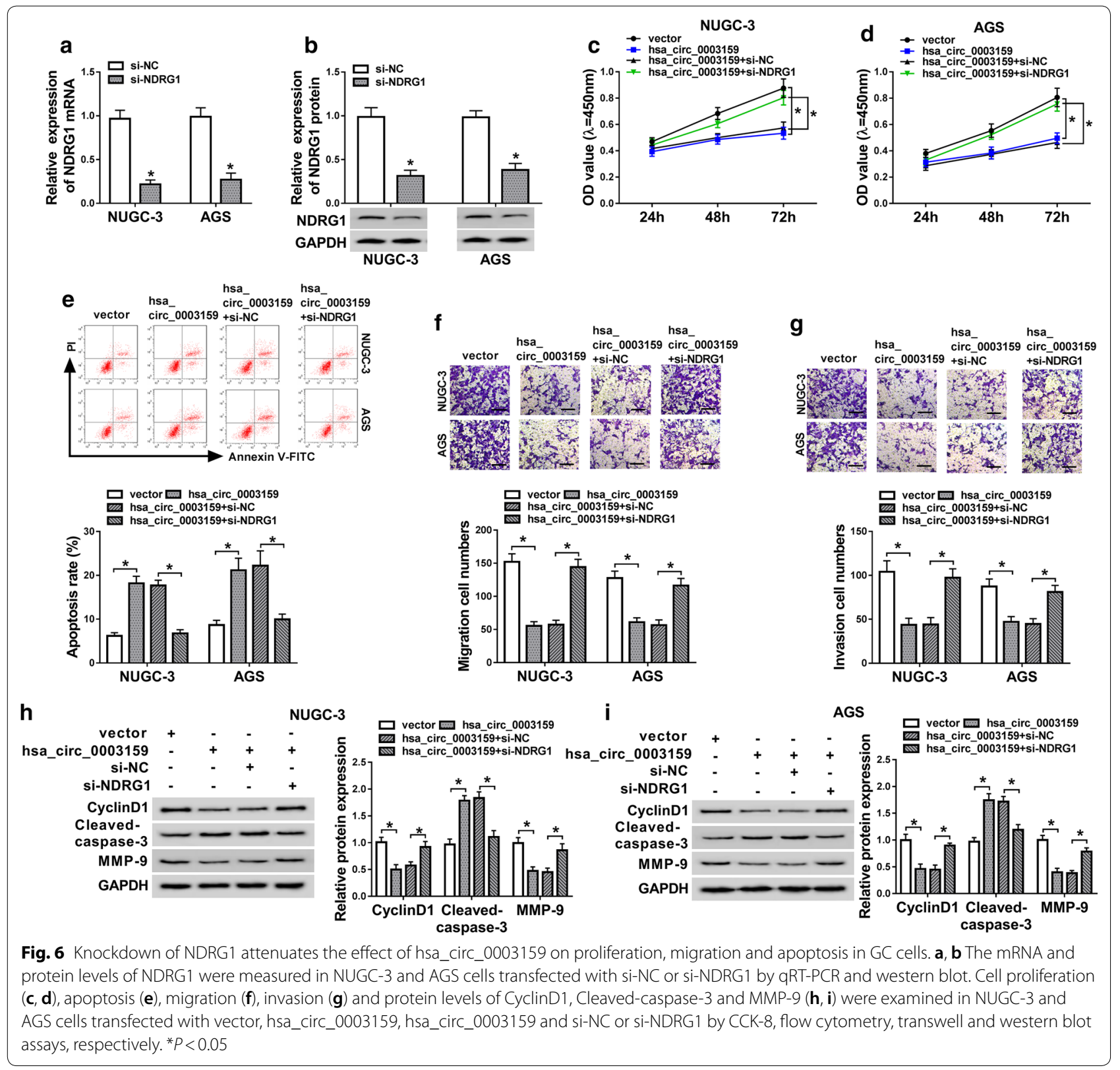

(Fig. 7a). Moreover, at the ending point, tumor weight of hsa_circ_0003159 group was lower than that in vector group (Fig. 7b). The hematoxylin-eosin staining showed that these were less malignant cells in hsa_circ_0003159 group than vector group (Fig. 7c). Additionally, the expression levels of hsa_circ_0003159, miR-223-3p and NDRG1 were detected in tumor tissues of each group. The results showed that the levels of hsa_circ_0003159 and NDRG1 were significantly increased but miR-223-3p expression was decreased in hsa_circ_0003159 group compared with those in vector group (Fig. $7 \mathrm{~d}-$ g). Furthermore, down-regulation of CyclinD1 and MMP-9 and elevation of Cleaved-caspase-3 were shown in hsa circ_0003159 group compared with that in vector group (Fig. 7h).

\section{Discussion}

$\mathrm{GC}$ is a public problem with high incidence and mortality [21]. CircRNAs have the critical clinical significance in GC [22]. The former work suggested that down-regulation of hsa_circ_0003159 had the potential clinical value in GC [10]. However, how and whether hsa_circ_0003159 mediated GC progression remain unclear. Here we found that hsa_circ_0003159 was lowly 


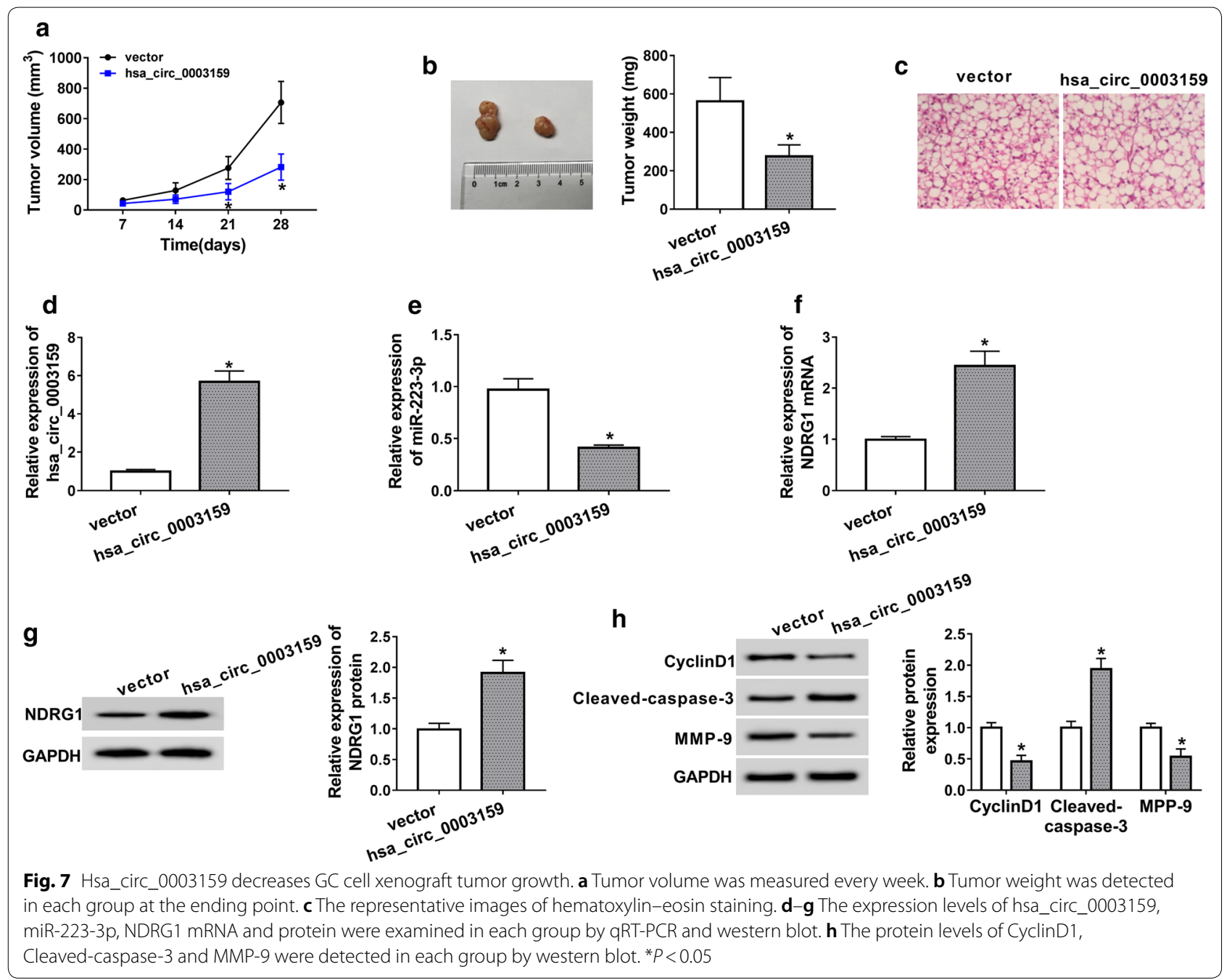

expressed and associated with poor outcome of patients in GC. CyclinD1 was a proliferation-associated marker of GC cells by promoting cell cycle process [23]. Caspase-3 activation mediated cell apoptosis in many cell lines in response to some stimuli [24]. MMP-9 activity was associated with the abilities of migration and invasion of GC cells [25]. By detecting their levels and performing the functional assays, we found that hsa circ_0003159 inhibited cell proliferation, migration and invasion but promoting apoptosis of GC cells, disclosing that hsa_circ_0003159 could be used as a tumor suppressor in GC. However, the underlying mechanism of hsa_circ_0003159 remains undetermined. In the current work, we were the first to confirm the ceRNA network of hsa_circ_0003159/miR-223-3p/NDRG1 in GC cells.

In this study, we found that hsa_circ_0003159 was mainly expressed in cytoplasm of GC cells, indicating that hsa_circ_0003159 could function as a sponge of miRNAs. Previous studies demonstrated that miR-223-3p played essential role in human cancers by promoting or inhibiting cancer development [12-14]. Here we first confirmed that hsa_circ_0003159 could sponged and negatively regulated miR-223-3p in GC cells. Moreover, we found that miR-223-3p expression was increased in $\mathrm{GC}$, indicating the high expression of miR-223-3p might contribute to the development of $\mathrm{GC}$, which was also in agreement with former works that displayed the proproliferation and pro-metastasis role of miR-223-3p in GC cells $[15,26]$. Similarly, we also found that up-regulation of miR-223-3p reversed the suppressive effect of hsa_circ_0003159 by its pro-cancer function. Meanwhile, these findings suggested that hsa_circ_0003159 repressed GC progression by sponging miR-223-3p in vitro.

Next, we further explored the ceRNA network mediated by hsa_circ_0003159 in GC cells. Having given that hsa_circ_0003159 could sponge miR-223-3p, we explored the targets of miR-223-3p and confirmed that NDRG1 as a target of miR-223-3p had the same binding 
sites with hsa_circ_0003159. In addition, we validated that hsa_circ_0003159 could promote NDRG1 expression by competitively binding to miR-223-3p, supporting the ceRNA of hsa_circ_0003159/miR-223-3p/NDRG1 in GC cells. NDRG1 has been regarded as a metastasis suppressor in human cancers [17]. Besides, the rescue experiments displayed that NDRG1 knockdown attenuated the suppressive effect of hsa_circ_0003159 on GC progression, indicating the inhibitive role of NDRG1 in GC, which was also in agreement with previous studies $[18,27]$. This also uncovered that hsa_circ_0003159 regulated GC development by targeting NDRG1. Moreover, xenograft model was widely used to analyze the pathogenesis of GC in vivo [28, 29]. Here we also established the murine xenograft model to validate the anti-cancer role of hsa_circ_0003159 and the regulatory network of hsa_circ_0003159/miR-223-3p/NDRG1 in vivo.

\section{Conclusion}

In conclusion, this study disclosed the anti-cancer role of hsa_circ_0003159 in GC through inhibiting proliferation, migration, invasion and xenograft tumor growth and promoting apoptosis, possibly by regulating miR-223-3p and NDRG1. This research indicated a new target for treatment of GC.

\section{Supplementary information}

Supplementary information accompanies this paper at https://doi. org/10.1186/s12935-020-1119-0.

Additional file 1: Figure S1. The effect of hsa_circ_0003159 on cell cycle in GC cells. The cell cycle distribution was detected in NUGC-3 and AGS cells transfected with vector or hsa_circ_0003159 by flow cytometry. ${ }^{*} P<0.05$.

Additional file 2: Figure S2. The effect of hsa_circ_0003159 on epithelialmesenchymal transition in GC cells. The expression levels of E-cadherin, $\mathrm{N}$-cadherin, Snail and Slug were detected in NUGC-3 and AGS cells transfected with vector or hsa_circ_0003159 by western blot. ${ }^{*} P<0.05$.

\section{Acknowledgements}

Not applicable.

\section{Authors' contributions}

JW and WL conceived and designed the experiments; ZL and XW performed the experiments; JB and YS contributed reagents/materials/analysis tools; JW and WL wrote the paper. All authors read and approved the final manuscript.

\section{Funding}

This study was supported by the Zhuhai City, Guangdong Province Department of Science and Technology [Grant No. 20191210E030076].

\section{Availability of data and materials}

The datasets used and/or analyzed during the current study are available from the corresponding author on reasonable request.

\section{Ethics approval and consent to participate}

The study protocol was designed and approved by the ethical committee of the Fifth Affiliated Hospital of Sun Yat-Sen University.
Consent for publication

Informed consent was obtained from all patients.

\section{Competing interests}

The authors declare that they have no competing interests.

\section{Author details}

${ }^{1}$ Department of Gastrointestinal Surgery, The Fifth Affiliated Hospital of Sun Yat-Sen University, No. 52, East Meihua Road, Zhuhai 519000, Guangdong, China. ${ }^{2}$ Department of Thoracic Oncology, The Fifth Affiliated Hospital of Sun Yat-Sen University, Zhuhai, China. ${ }^{3}$ Department of General Surgery, The Fifth Affiliated Hospital of Sun Yat-Sen University, Zhuhai, China.

Received: 13 October 2019 Accepted: 20 January 2020

Published online: 19 February 2020

References

1. Van Cutsem E, Sagaert X, Topal B, Haustermans K, Prenen H. Gastric cancer. Lancet. 2016;388(10060):2654-64.

2. Rugge M, Genta RM, Di Mario F, El-Omar EM, El-Serag HB, Fassan M, et al. Gastric cancer as preventable disease. Clin Gastroenterol Hepatol. 2017;15(12):1833-43.

3. Necula L, Matei L, Dragu D, Neagu Al, Mambet C, Nedeianu S, et al. Recent advances in gastric cancer early diagnosis. World J Gastroenterol. 2019;25(17):2029-44.

4. Matsuoka T, Yashiro M. Biomarkers of gastric cancer: current topics and future perspective. World J Gastroenterol. 2018;24(26):2818-32.

5. Lei B, Tian Z, Fan W, Ni B. Circular RNA: a novel biomarker and therapeutic target for human cancers. Int J Med Sci. 2019;16(2):292-301.

6. Kristensen LS, Andersen MS, Stagsted LVW, Ebbesen KK, Hansen TB, Kjems J. The biogenesis, biology and characterization of circular RNAs. Nat Rev Genet. 2019;20(11):675-91.

7. Wang KW, Dong M. Role of circular RNAs in gastric cancer: recent advances and prospects. World J Gastrointest Oncol. 2019;1 1(6):459-69.

8. Huang S, Zhang X, Guan B, Sun P, Hong CT, Peng J, et al. A novel circular RNA hsa_circ_0008035 contributes to gastric cancer tumorigenesis through targeting the miR-375/YBX1 axis. Am J Transl Res. 2019;11(4):2455-62.

9. Liang M, Huang G, Liu Z, Wang Q, Yu Z, Liu Z, et al. Elevated levels of hsa_circ_006100 in gastric cancer promote cell growth and metastasis via miR-195/GPRC5A signalling. Cell Prolif. 2019;52(5):e12661.

10. Tian M, Chen R, Li T, Xiao B. Reduced expression of circRNA hsa_ circ_0003159 in gastric cancer and its clinical significance. J Clin Lab Anal. 2018;32(3):e22281.

11. Zhong Y, Du Y, Yang X, Mo Y, Fan C, Xiong F, et al. Circular RNAs function as ceRNAs to regulate and control human cancer progression. Mol Cancer. 2018;17(1):79.

12. Ding Q, Shen L, Nie X, Lu B, Pan X, Su Z, et al. MiR-223-3p overexpression inhibits cell proliferation and migration by regulating inflammation-associated cytokines in glioblastomas. Pathol Res Pract. 2018;214(9):1330-9.

13. Xiao W, Wang X, Wang T, Xing J. MiR-223-3p promotes cell proliferation and metastasis by downregulating SLC4A4 in clear cell renal cell carcinoma. Aging (Albany NY). 2019;11(2):615-33.

14. Chai B, Guo Y, Cui X, Liu J, Suo Y, Dou Z, et al. MiR-223-3p promotes the proliferation, invasion and migration of colon cancer cells by negative regulating PRDM1. Am J Transl Res. 2019;11(7):4516-23.

15. Li X, Zhang Y, Zhang H, Liu X, Gong T, Li M, et al. miRNA-223 promotes gastric cancer invasion and metastasis by targeting tumor suppressor EPB41L3. Mol Cancer Res. 2011;9(7):824-33.

16. Li J, Guo Y, Liang X, Sun M, Wang G, De W, et al. MicroRNA-223 functions as an oncogene in human gastric cancer by targeting FBXW7/hCdc4. J Cancer Res Clin Oncol. 2012;138(5):763-74.

17. Park KC, Paluncic J, Kovacevic Z, Richardson DR. Pharmacological targeting and the diverse functions of the metastasis suppressor, NDRG1, in cancer. Free Radic Biol Med. 2019.

18. Chang X, Xu X, Ma J, Xue X, Li Z, Deng P, et al. NDRG1 expression is related to the progression and prognosis of gastric cancer patients through 
modulating proliferation, invasion and cell cycle of gastric cancer cells. Mol Biol Rep. 2014;41(9):6215-23.

19. Dong X, Hong Y, Sun H, Chen C, Zhao X, Sun B. NDRG1 suppresses vasculogenic mimicry and tumor aggressiveness in gastric carcinoma. Oncol Lett. 2019;18(3):3003-16.

20. Livak KJ, Schmittgen TD. Analysis of relative gene expression data using real-time quantitative PCR and the 2(-Delta Delta C(T)) Method. Methods. 2001;25(4):402-8.

21. Thrift AP, El-Serag HB. Burden of gastric cancer. Clin Gastroenterol Hepatol. 2019. https://doi.org/10.1016/j.cgh.2019.07.045.

22. Shan C, Zhang Y, Hao X, Gao J, Chen X, Wang K. Biogenesis, functions and clinical significance of circRNAs in gastric cancer. Mol Cancer. 2019:18(1):136.

23. Yang L, Yu Y, Zhang Q, Li X, Zhang C, Mao T, et al. Anti-gastric cancer effect of Salidroside through elevating miR-99a expression. Artif Cells Nanomed Biotechnol. 2019;47(1):3500-10.

24. Nagata S. Apoptosis and clearance of apoptotic cells. Annu Rev Immunol. 2018;36:489-517.

25. Wang M, Tian Z, Zhu Y, Ding J, Li C, Zhou Y, et al. Sichong formula inhibits the proliferation and migration of human gastric cancer cells. Onco Targets Ther. 2019;12:5741-50.
26. Ma L, Chen Y, Zhang B, Liu G. Increased microRNA-223 in Helicobacter pylori-associated gastric cancer contributed to cancer cell proliferation and migration. Biosci Biotechnol Biochem. 2014;78(4):602-8.

27. Chang $X, X u X, X u e X, M a ~ J$, Li Z, Deng P, et al. NDRG1 controls gastric cancer migration and invasion through regulating MMP-9. Pathol Oncol Res. 2016;22(4):789-96.

28. Xuan Y, Wang Y. Long non-coding RNA SNHG3 promotes progression of gastric cancer by regulating neighboring MED18 gene methylation. Cell Death Dis. 2019;10(10):694.

29. Li X, Luo Y, Liu L, Cui S, Chen W, Zeng A, et al. The long noncoding RNA ZFAS1 promotes the progression of glioma by regulating the miR150-5p/PLP2 axis. J Cell Physiol. 2020;235(3):2937-46.

\section{Publisher's Note}

Springer Nature remains neutral with regard to jurisdictional claims in published maps and institutional affiliations.
Ready to submit your research? Choose BMC and benefit from:

- fast, convenient online submission

- thorough peer review by experienced researchers in your field

- rapid publication on acceptance

- support for research data, including large and complex data types

- gold Open Access which fosters wider collaboration and increased citations

- maximum visibility for your research: over $100 \mathrm{M}$ website views per year

At BMC, research is always in progress.

Learn more biomedcentral.com/submissions 